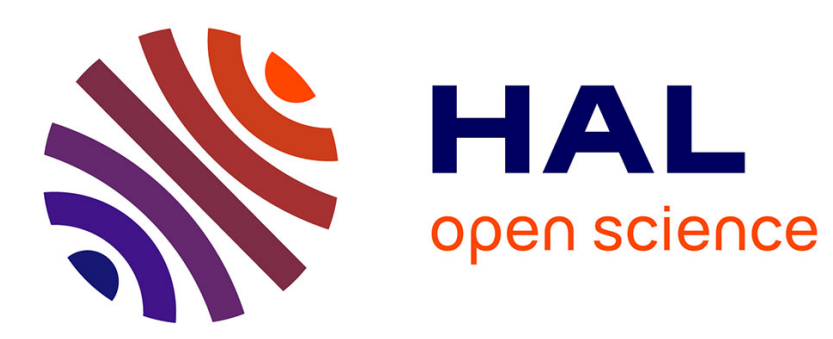

\title{
Mesure directe de la permittivité complexe de matériaux à faibles pertes dans une gamme de fréquences inférieure au Hertz
}

S. Dinculescu, Thierry Lebey, A. Loubière, Bui Ai

\section{- To cite this version:}

S. Dinculescu, Thierry Lebey, A. Loubière, Bui Ai. Mesure directe de la permittivité complexe de matériaux à faibles pertes dans une gamme de fréquences inférieure au Hertz. Journal de Physique III, 1996, 6 (8), pp.991-1004. 10.1051/jp3:1996167 • jpa-00249512

\section{HAL Id: jpa-00249512 https://hal.science/jpa-00249512}

Submitted on 1 Jan 1996

HAL is a multi-disciplinary open access archive for the deposit and dissemination of scientific research documents, whether they are published or not. The documents may come from teaching and research institutions in France or abroad, or from public or private research centers.
L'archive ouverte pluridisciplinaire HAL, est destinée au dépôt et à la diffusion de documents scientifiques de niveau recherche, publiés ou non, émanant des établissements d'enseignement et de recherche français ou étrangers, des laboratoires publics ou privés. 


\title{
Mesure directe de la permittivité complexe de matériaux à faibles pertes dans une gamme de fréquences inférieure au Hertz
}

\author{
S. Dinculescu, T. Lebey $\left({ }^{*}\right)$, A. Loubière et Bui Ai \\ Laboratoire de Génie Électrique de Toulouse $\left({ }^{* *}\right), 118$ Route de Narbonne, \\ 31062 Toulouse Cedex, France
}

(Reçu le 16 février 1996, révisé et accepté le 22 mai 1996)

PACS.77. - Dielectrics, piezoelectrics and ferroelectrics and their properties
PACS.07.50.-e - Electrical and electronic components, instruments, and techniques
PACS.81.70.Cv - Nondestructive testing ultrasonic testing, photoacoustic testing

Résumé. - La nécessité d'une caractérisation diélectrique précise dans la gamme de fréquences inférieures au $\mathrm{Hz}$, et les limites des systèmes actuels nous ont conduits à proposer et à développer un dispositif permettant d'effectuer une mesure directe et automatisée de la permittivité. La mise au point de ce système est décrite et ses limitations sont données. Des résultats obtenus sur différents types de matériaux diélectriques solides sont présentés et discutés.

\begin{abstract}
Very low frequency characterisation appears as a key point for dielectric material understanding. Nevertheless, up to now, the different methods available have some limitations whichunable accurate measurements. We propose in the following a new experimental set-up allowing permittivity determination. Different solid dielectric materials have been studied and the results are presented and discussed.
\end{abstract}

\section{Introduction}

La connaissance précise du comportement des matériaux diélectriques semble nécessaire pour assurer d'une part un fonctionnement optimal et d'autre part la maintenance efficace des équipements et des systèmes dont ils constituent en général les parties les plus faibles $[1,2]$. L'étude de l'évolution de leurs caractéristiques initiales, pendant et après l'application de contraintes de différentes natures, constitue un axe de recherche important en Génie Électrique. Cette caractérisation peut être, ou non, de nature destructive $[3,4]$. Pour des raisons évidentes, les méthodes non destructives suscitent la plus grande attention. Elles offrent en effet, la possibilité d'effectuer le suivi du comportement des systèmes sans les endommager. De nombreuses grandeurs physiques sont susceptibles d'être utilisées $[5,6]$. Parmi elles, la permittivité diélectrique semble devoir être retenue. Elle permet d'obtenir des informations sur les propriétés intrinsèques des matériaux (en fonction de leur structure, de leur composition,..) [7] ou sur leur vieillissement [8], à condition que son évolution soit étudiée sur une large gamme de fréquence. Toutefois, si sa mesure ne présente quasiment plus de problème dans la gamme des

${ }^{*}$ ) Auteur auquel doit être adressée la correspondance (e-mail: lebey@lget.ups-tlse.fr)

$\left({ }^{* *}\right)$ UA CNRS 304

(C) Les Éditions de Physique 1996 
fréquences intermédiaires (de $10 \mathrm{~Hz}$ à $1 \mathrm{MHz}$ ), il n'en est plus de même de part et d'autre de ces limites. Pour les hautes fréquences, nous avons proposé récemment deux cellules permettant une caractérisation diélectrique de $10 \mathrm{MHz}$ à $6 \mathrm{GHz}$ [9]. L'intérêt actuel pour les mesures à très basse fréquence est justifié, quant à lui, par les informations fournies tant d'un point de vue théorique que pratique. D'une part, ces caractérisations s'avèrent primordiales pour l'étude et la modélisation des mécanismes de polarisation (d'orientation, interfaciale, par charge d'espace... ). D'autre part, l'analyse de ces phénomènes peut contribuer à la compréhension du comportement des matériaux diélectriques en fonction de leurs constituants, mais aussi lors de la modélisation de certains de leurs mécanismes de vieillissement. Nous présentons dans ce qui suit, un dispositif permettant une caractérisation directe de matériaux à faibles pertes dans la gamme des très basses fréquences (de $10^{-3}$ à $1 \mathrm{~Hz}$ ). Nous rappellerons tout d'abord le principe des mesures permettant actuellement cette caractérisation et nous donnerons leurs limites. Cette présentation nous permettra d'expliquer les raisons de notre approche. La mise en œuvre du dispositif sera alors décrite tant du point de vue théorique que pratique. Enfin, après une phase de validation, des exemples d'application à différents matériaux diélectriques solides seront donnés et leurs résultats seront discutés.

\section{Principes de mesure}

Deux grandes catégories de méthodes sont actuellement susceptibles de permettre une caractérisation dans la gamme des très basses fréquences [10]. Il s'agit des méthodes dans le domaine temporel (dites méthodes indirectes) et dans le domaine fréquentiel (dites méthodes directes). Dans le premier cas, un échelon de tension est appliqué aux bornes de l'échantillon étudié et sa réponse, c'est-à-dire le courant, est enregistrée en fonction du temps. Ces données sont alors transférées du domaine temporel au domaine fréquentiel, soit par une transformation de Fourier, soit, dans le cadre de certaines approximations concernant la décroissance du courant, par une transformation dite de Hamon [11]. Cette méthode, dont le principe est très simple, présente toutefois toute une série d'inconvénients. Tout d'abord, pour que l'ensemble des mécanismes de polarisation soit décrit, il faut que tous les phénomènes de relaxation qui peuvent contribuer à la valeur finale du courant soient achevés à l'arrêt de l'acquisition du courant. Cela ne se produit qu'au bout d'un temps $t_{c}$, souvent difficile à apprécier. Pour déterminer le minimum de $t_{c}$, une méthode consiste à charger et à décharger le condensateur formé par le diélectrique avec des temps de charge croissants, jusqu'à ce que la valeur du courant de décharge à un moment $t_{\mathrm{d}}$ devienne indépendante de $t_{\mathrm{c}}$. Cette expérience est nécessaire pour assurer l'équivalence entre la réponse analysée et la réponse vraie du diélectrique [12]. Ensuite, une fois cette fonction réponse obtenue, il est nécessaire de calculer la transformée de Fourier des données ce qui suppose une acquisition avec une période d'échantillonnage bien précise. Dans ces conditions, on rencontre quand même des difficultés à apprécier correctement les limites de validité en fréquence de la transformée de Fourier. Enfin, cette méthode nécessite l'utilisation de systèmes de mesure de courant très performants. Ces derniers doivent présenter à la fois une sensibilité élevée et une dérive faible. L'ensemble de ces contraintes fait que ce type de caractérisation, si elle est très largement admise d'un point de vue conceptuel, n'est que très rarement utilisée dans des conditions suffisantes pour ne pas remettre en doute ses résultats. Un article de synthèse [13] résume bien cette problématique.

Les méthodes directes ont comme point de départ le schéma équivalent du condensateur réel, modélisé par une capacité idéale en parallèle avec une résistance. Le but de ces mesures est de déterminer les valeurs de ces composants en utilisant diverses configurations de ponts. Une des configurations les plus connues est le pont de Schering, schématisé sur la figure 1. La cellule contient l'échantillon à tester (représenté par $R_{x}$ et $C_{x}$ ) et la mesure proprement dite 


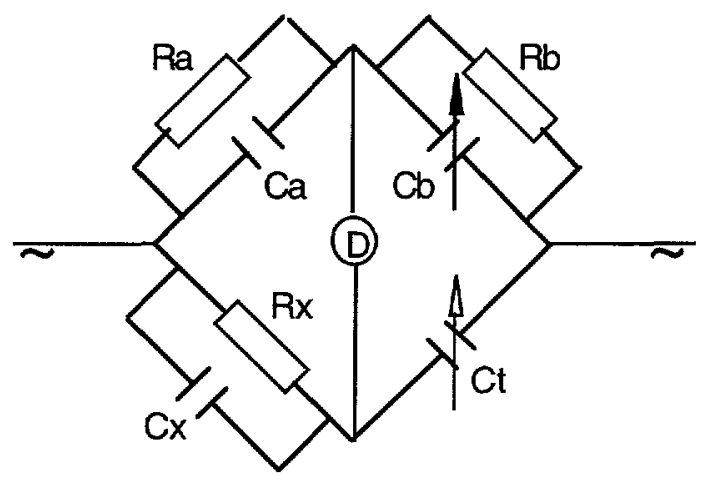

Fig. 1. - Configuration d'un pont de type Schering.

[Schering bridge set-up.]

consiste à équilibrer le pont l'aide des éléments variables $C_{\mathrm{b}}$ et $C_{t}$. Les valeurs de $R_{x}$ et $C_{x}$ peuvent être déterminées soit directement (connaissant les valeurs des autres paramètres), soit par une méthode de substitution. Le principal désavantage de cette méthode est que lorsque I'on tend vers les très basses fréquences, l'ensemble des éléments utilisés peut ne pas avoir les comportements escomptés.

Une autre approche consiste à mesurer les composantes de la tension en phase et en quadrature avec la tension appliquée aux bornes de l'échantillon $\left(U_{1}\right)$ et à faire ensuite la même chose pour une résistance connectée en série $\left(U_{2}\right)$ (Fig. 2). Les valeurs des paramètres du schéma équivalent peuvent alors être déterminées en connaissant la valeur de la résistance. Ce type de mesure est réalisé à l'aide d'un analyseur de gain et de phase. Cependant, la sensibilité des dispositifs actuels (quelques dizaines de $\mu \mathrm{V}$ ) et les limitations imposées par la valeur des impédances d'entrée et celle présente entre les deux voies, font que si cette méthode est très largement utilisée en électrochimie ou pour des matériaux dont l'impédance équivalente est faible, elle ne peut être appliquée telle qu'elle sur les matériaux diélectriques solides les plus souvent rencontrés en Génie Électrique. Nous présentons donc une variante permettant d'utiliser tous les avantages de cette méthode sans ses inconvénients.

\section{Dispositif proposé et mise en œuvre}

Le dispositif que nous avons mis au point repose sur l'utilisation d'un analyseur de gain et de phase (Solartron 1253) et d'un amplificateur conçu et réalisé au Laboratoire. L'analyseur de gain et de phase est constitué d'un générateur, qui peut délivrer un signal sinusoïdal d'amplitude et de fréquence variables $\left(0<U<10 V_{\text {efficace, }} 10^{-3}<f<2 \times 10^{4} \mathrm{~Hz}\right)$ et de deux analyseurs capables de déterminer les composantes en phase (mesure de $a$ ) et en quadrature (mesure de b) de la tension, en prenant comme référence le signal délivré par le générateur. La détermination de ces composantes est effectuée par une méthode de corrélation (Fig. 3). Cette méthode de calcul présente l'avantage de limiter l'influence du bruit car, après intégration, sa contribution au résultat final devient d'autant moins importante que le nombre de périodes d'intégration est grand. Le diagramme global du circuit de mesure utilisé pour la détermination des valeurs des composantes du schéma équivalent d'un échantillon est donné sur la figure 4 , où $R_{x}, C_{x}$ sont les valeurs à déterminer (caractéristiques du matériau étudié), $R_{\mathrm{d}}$ et $C_{\mathrm{d}}$ sont la résistance de détection et sa capacité parasite et $a+j b$ et $A+j B$ sont les valeurs affichées par 


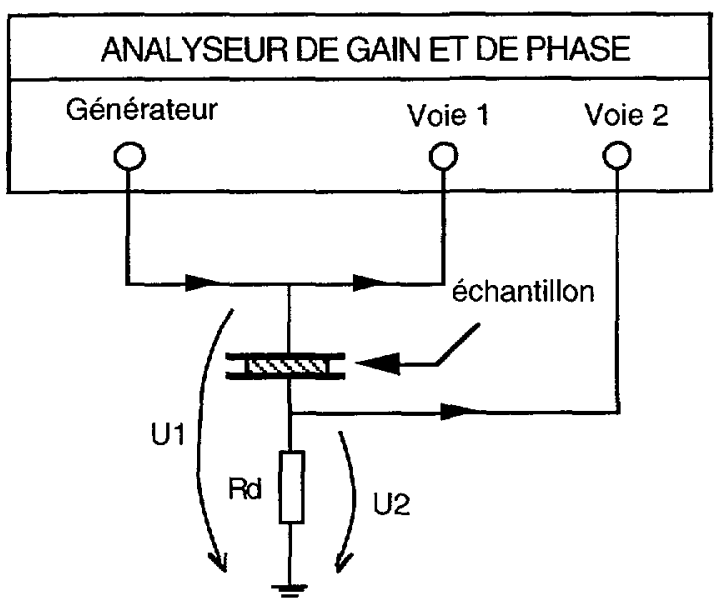

Fig. 2. - Schéma de principe des mesures utilisant la méthode fréquentielle directe. [Principle set-up for direct frequency measurements.]

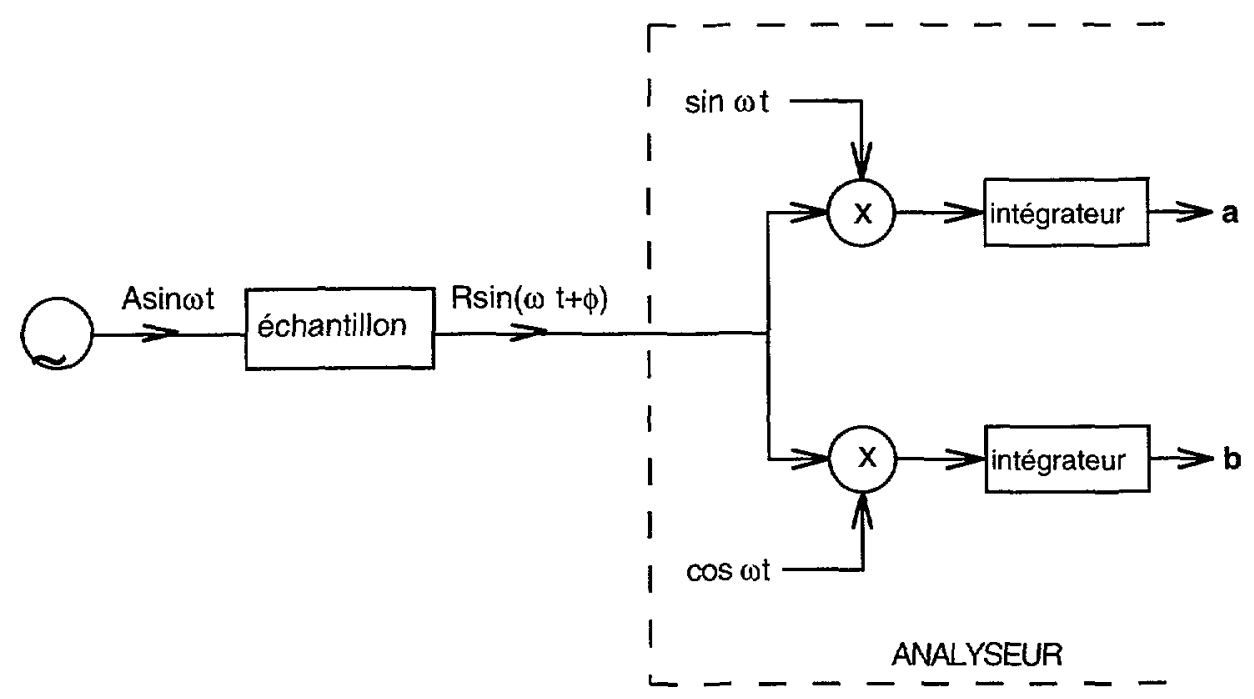

Fig. 3. -- Principe des méthodes de corrélation.

[Principle of correlation methods.]

l'analyseur après la mesure. Avec ces notations, les valeurs $C_{x}$ et $R_{x}$ peuvent être calculées à l'aide des relations suivantes [14] :

$$
C_{x}=\frac{1}{\omega R_{\mathrm{d}}}\left[\frac{b A-a B}{(A-a)^{2}+(B-b)^{2}}\right]+C_{\mathrm{d}}\left[\frac{a(A-a)+b(B-b)}{(A-a)^{2}+(B-b)^{2}}\right]
$$

et

$$
\frac{1}{R_{x}}=\frac{1}{R_{\mathrm{d}}}\left[\frac{a(A-a)+b(B-b)}{(A-a)^{2}+(B-b)^{2}}\right]-\omega C_{\mathrm{d}}\left[\frac{b A-a B}{(A-a)^{2}+(B-b)^{2}}\right] .
$$




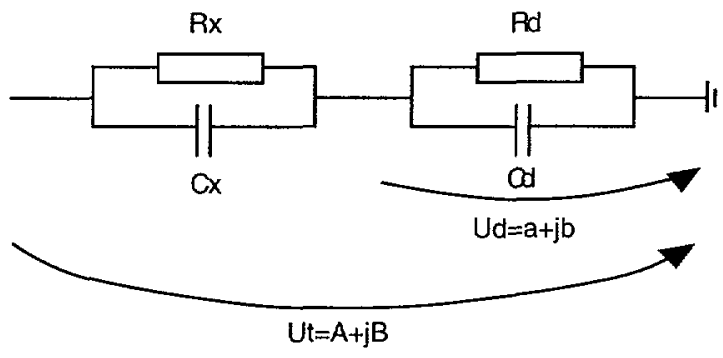

Fig. 4. -- Configuration du circuit de mesure.

[Measuring circuit configuration.]

Comme nous l'avons signalé précédemment, l'utilisation directe de ce dispositif est rendue quasi-impossible aussitôt que l'on s'intéresse à des matériaux sans pertes ou à pertes peu élevées. L'impédance de ces matériaux est alors telle que deux problèmes peuvent surgir. Tout d'abord, une valeur assez faible de la résistance de détection va être choisie afin que la plus grande partie de la tension appliquée aux bornes du circuit complet (échantillon en série avec la résistance de détection) se retrouve aux bornes de l'échantillon. Cependant une valeur trop faible conduit à l'impossibilité de détecter correctement les valeurs $a$ et $b$ compte tenu de la sensibilité limitée des circuits de l'analyseur. Pour obtenir une valeur correcte de ces grandeurs, $R_{\mathrm{d}}$ doit donc être augmentée mais dans une certaine limite au delà de laquelle un deuxième problème, associé à la valeur de l'impédance d'entrée de l'analyseur (1 M $\mathrm{M})$, est rencontré.

Signalons enfin, une dernière limitation très rarement mentionnée. Des valeurs quasiment identiques pour les deux tensions $U_{\mathrm{d}}$ et $U_{\mathrm{t}}$ sont, en effet, obtenues lorsqu'un échantillon d'impédance très élevée est étudié. Les caractéristiques du matériau ne peuvent alors plus être déterminées car son impédance équivalente est court-circuitée par l'impédance présente entre les deux voies de l'analyseur, dont la valeur a été déterminée à $330 \mathrm{M} \Omega$. Pour des échantillons dont l'impédance équivalente est égale ou supérieure à cette valeur, la mesure devient fortement erronée. Cette dernière limitation est à notre avis la plus critique de ce type d'appareillage.

Afin de contourner l'ensemble de ces problèmes, revenons à la configuration de base des mesures effectuées à l'aide d'un pont (Fig. 5). Le principe de leur fonctionnement repose sur l'obtention d'un équilibre. Ce dernier offre alors la possibilité de déterminer une impédance inconnue en effectuant une comparaison avec une référence de valeur connue. Les tensions $V_{x}$ et $V_{\mathrm{r}}$ ont la même amplitude mais sont en opposition de phase. Afin d'obtenir un équilibre, on agit sur l'impédance $Y_{\mathrm{r}}$ jusqu'à ce que la tension $V_{\mathrm{d}}$ s'annule, ce qui se traduit par l'égalité des deux courants $I_{x}$ et $I_{\mathrm{r}}$. Notons toutefois que la mesure de la tension aux bornes de $R_{\mathrm{d}}$ n'est qu'une des nombreuses possibilités pour déterminer la différence entre les deux courants. Une autre approche consiste à mesurer directement ce courant de différence $I_{\mathrm{d}}$ sans introduire de résistance de détection. Il suffit pour cela de maintenir le potentiel du point $S$ à un niveau aussi proche de zéro que possible, afin que la quasi-totalité de la tension $V_{x}$ soit appliquée aux bornes de l'échantillon. Un amplificateur opérationnel peut assurer ces deux fonctions (mesure du courant de différence $I_{\mathrm{d}}$ et maintien du point $S$ au potentiel virtuel de la masse). Le bras qui contient la référence peut même être supprimé. On ne se contente plus de voir si l'équilibre est atteint pour déterminer par identification $Y_{x}$, mais on procède à sa détermination directe à l'aide de l'amplificateur. Le schéma correspondant à cette configuration est alors représenté 


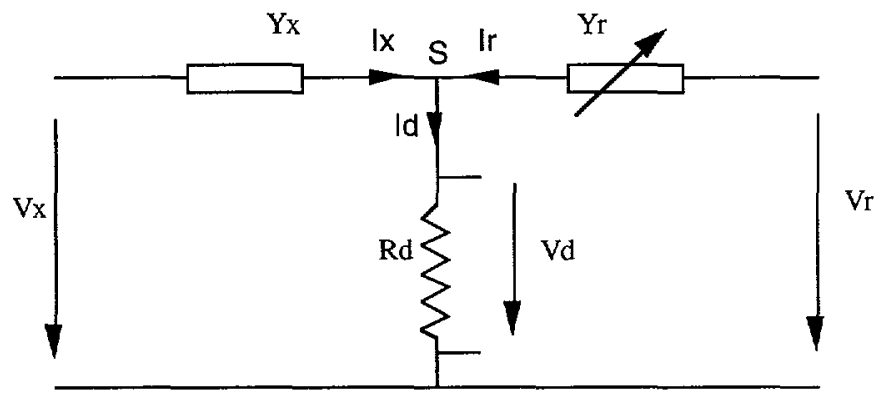

Fig. 5. - Schéma de principe d'un pont de mesure.

[Principle of bridge measurements.]

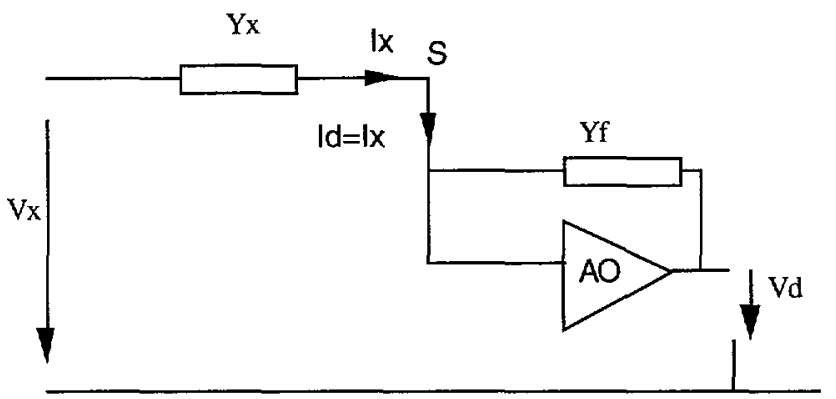

Fig. 6. - Schéma du circuit de mesure modifié.

[Configuration of the modified measuring arrangement.]

sur la figure 6. Pour un amplificateur idéal le courant va être donné par :

$$
I_{\mathrm{d}}=-V_{\mathrm{d}} Y_{\mathrm{f}}
$$

où

$$
V_{\mathrm{d}}=-V_{x} \frac{Y_{x}}{Y_{\mathrm{f}}}
$$

est la tension de sortie de l'amplificateur opérationnel et $Y_{\mathrm{f}}$ la valeur de l'élément de contreréaction.

Comment doit on alors choisir $Y_{\mathrm{f}}$ ? La première solution consiste à utiliser une résistance pure, $R_{\mathrm{f}}$ de valeur suffisamment élevée pour que la tension $V_{\mathrm{d}}$ soit d'amplitude acceptable. Dans ce cas, les relations qui permettent de calculer les paramètres de l'échantillon $Y_{x}$, assimilé à un condensateur plan de capacité géométrique $C_{0}$ entre les armatures duquel est placé un diélectrique caractérisé par sa permittivité relative complexe $\varepsilon^{*}$ (définie par $\varepsilon^{*}=\varepsilon^{\prime}-j \varepsilon^{\prime \prime}$ ) conduisent à $Y_{x}=j \omega C_{0}\left(\varepsilon^{\prime}-\jmath \varepsilon^{\prime \prime}\right)$. En séparant les parties réelle et imaginaire de la relation (2), deux égalités peuvent être écrites à partir desquelles les valeurs de $\varepsilon^{\prime}$ et $\varepsilon^{\prime \prime}$ seront déduites :

$$
\begin{aligned}
\varepsilon^{\prime} & =\frac{-\operatorname{Im}\left(V_{\mathrm{d}}\right)}{\omega V_{x} R_{\mathrm{f}} C_{0}} \\
\varepsilon^{\prime \prime} & =\frac{-\operatorname{Re}\left(V_{\mathrm{d}}\right)}{\omega V_{x} R_{\mathrm{f}} C_{0}}
\end{aligned}
$$


où $\operatorname{Re}\left(V_{\mathrm{d}}\right)$ et $\operatorname{Im}\left(V_{\mathrm{d}}\right)$ sont respectivement les parties réelle et imaginaire de la tension de sortie $V_{\mathrm{d}}$. On peut alors constater que, si d'un point de vue théorique les relations de $\varepsilon^{\prime}$ et $\varepsilon^{\prime \prime}$ sont simples à utiliser, la sensibilité du détecteur compte tenu du gain du montage, diminue avec la fréquence $\left(V_{\mathrm{d}}\right.$ est proportionnelle à $\left.\omega\right)$. D'un point de vue pratique, pour des raisons de stabilité de la boucle de contre-réaction, il faut connecter une capacité auxiliaire $C_{\mathrm{f}}$ en parallèle avec $R_{\mathrm{f}}$. L'admittance de contre-réaction n'est plus $1 / R_{\mathrm{f}}$ mais $\left(1 / R_{\mathrm{f}}\right)+j \omega C_{\mathrm{f}}$ et la simplicité des relations qui précédent disparaît. Une autre solution doit donc être trouvée. Elle consiste à utiliser comme élément de contre-réaction une capacité $C_{\mathrm{f}}$. Les relations (3) deviennent alors :

$$
\begin{aligned}
\varepsilon^{\prime} & =\frac{-\operatorname{Re}\left(V_{\mathrm{d}}\right) C_{\mathrm{f}}}{V_{x} C_{0}} \\
\varepsilon^{\prime \prime} & =\frac{\operatorname{Im}\left(V_{\mathrm{d}}\right) C_{\mathrm{f}}}{V_{x} C_{0}}
\end{aligned}
$$

et la sensibilité de la mesure ne dépend plus de la fréquence. Cependant, d'autres inconvénients apparaissent. L'élément de contre-réaction étant purement capacitif, il n'y a plus de voie de courant continu entre l'entrée et la sortie de l'amplificateur opérationnel. La tension de sortie $V_{\mathrm{d}}$ est soumise à une dérive positive, due à la présence d'un courant de décalage, pouvant conduire à la saturation de l'amplificateur. La solution consiste alors à effectuer la mesure avant que celle-ci ne soit atteinte. Il faut donc choisir un amplificateur qui présente un courant de décalage le plus faible possible et prendre des précautions au niveau de la réalisation pratique du montage. Nous n'entrerons pas dans le détail de celles-ci qui sont en général assez bien décrites dans les catalogues des constructeurs [15]. Nous avons enfin apporté une amélioration qui consiste à décharger de manière automatique, entre deux mesures, le condensateur placé en contre-réaction.

Le choix de la méthode de mesure effectué, l'ensemble expérimental a été entièrement automatisé en raison de la durée importante nécessaire à une caractérisation diélectrique dans la gamme des fréquences les plus basses. Nous présentons dans ce qui suit les résultats que ce dispositif permet d'obtenir sur différents matériaux.

\section{Résultats expérimentaux}

4.1. Validation DU Dispositif. - Tout d'abord, afin de valider le dispositif mis au point, des modèles du comportement électrique de matériaux diélectriques sont étudiés. La majorité des mécanismes de relaxation suivant des lois de type Debye plus ou moins distribuées, l'étude de la réponse de circuits $R C$ pour différentes valeurs de $R$ et de $C$ est entreprise. Le choix de ces valeurs est fait pour que la fréquence de relaxation à obtenir se situe dans la gamme des fréquences les plus faibles. Pour ces raisons, différentes valeurs du couple $(R, C)$ sont retenues. Les résultats obtenus pour deux d'entre elles sont représentés sur les figures 7 et 8 . Une bonne concordance entre les valeurs mesurées et calculées est obtenue. Notons toutefois que dans le domaine des fréquences les plus élevées, une divergence apparaît. Celle-ci est liée à la bande passante de l'amplificateur utilisé, insuffisamment large pour couvrir tout le spectre de fréquence du générateur. Cette limitation n'en est cependant pas vraiment une puisqu'elle se produit dans une gamme de fréquence où des dispositifs classiques peuvent être utilisés. Nous pouvons donc conclure de ces tests que le système de mesure proposé présente un comportement suffisamment correct pour que différents types de matériaux diélectriques solides, représentatifs de ceux rencontrés en Génie Électrique soient étudiés. 

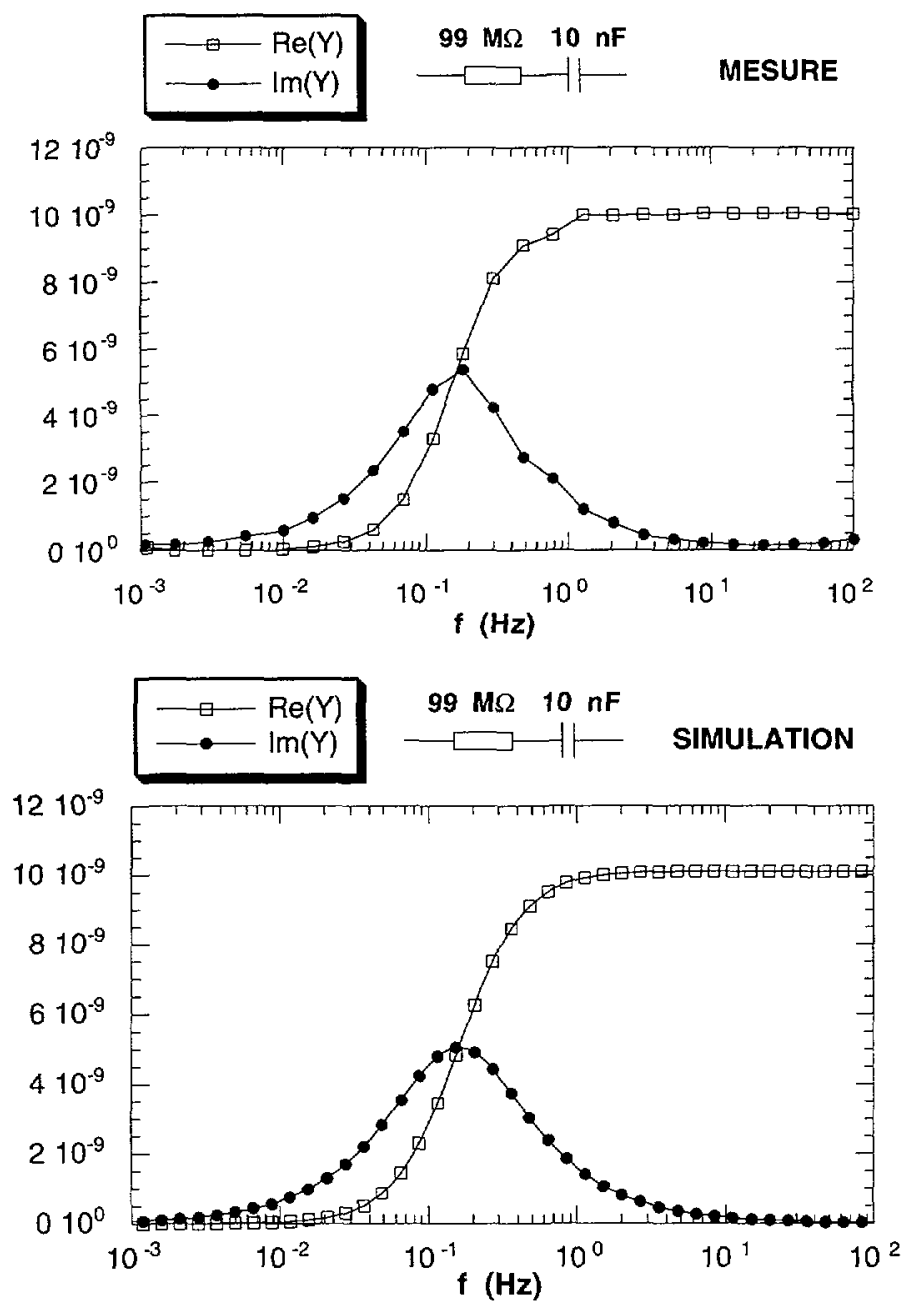

Fig. 7. - Admittance complexe en fonction de la fréquence pour un échantillon modèle : $R=99 \mathrm{M} \Omega$, $C=10 \mathrm{nF}$.

[Complex admittance vs. frequency for a model sample: $R=99 \mathrm{M} \Omega, C=10 \mathrm{nF}$.]

Afin d'être le plus complet possible, nous avons choisi d'étudier la réponse diélectrique de deux catégories de matériaux : d'une part, des céramiques à base d'oxyde de zinc en fonction de leur composition et pour une composition identique en fonction de la température, et d'autre part des matériaux polymères.

4.2. CARACTÉRISATION DES CÉRAMIQUES À BASE D'OXYDE DE ZINC. - Ces matériaux, dont l'impédance présente une non-linéarité avec la tension appliquée, les rend très utiles pour la réalisation d'éléments de protection contre les surtensions. Du point de vue structurel, ces céramiques sont composées de grains de $\mathrm{ZnO}$ (dopés n) et de joints de grains (riches en oxyde de Bismuth). Cette structure inhomogène permet l'apparition de mécanismes de polarisation interfaciale. Ces mécanismes caractéristiques de matériaux constitués de plusieurs phases de 

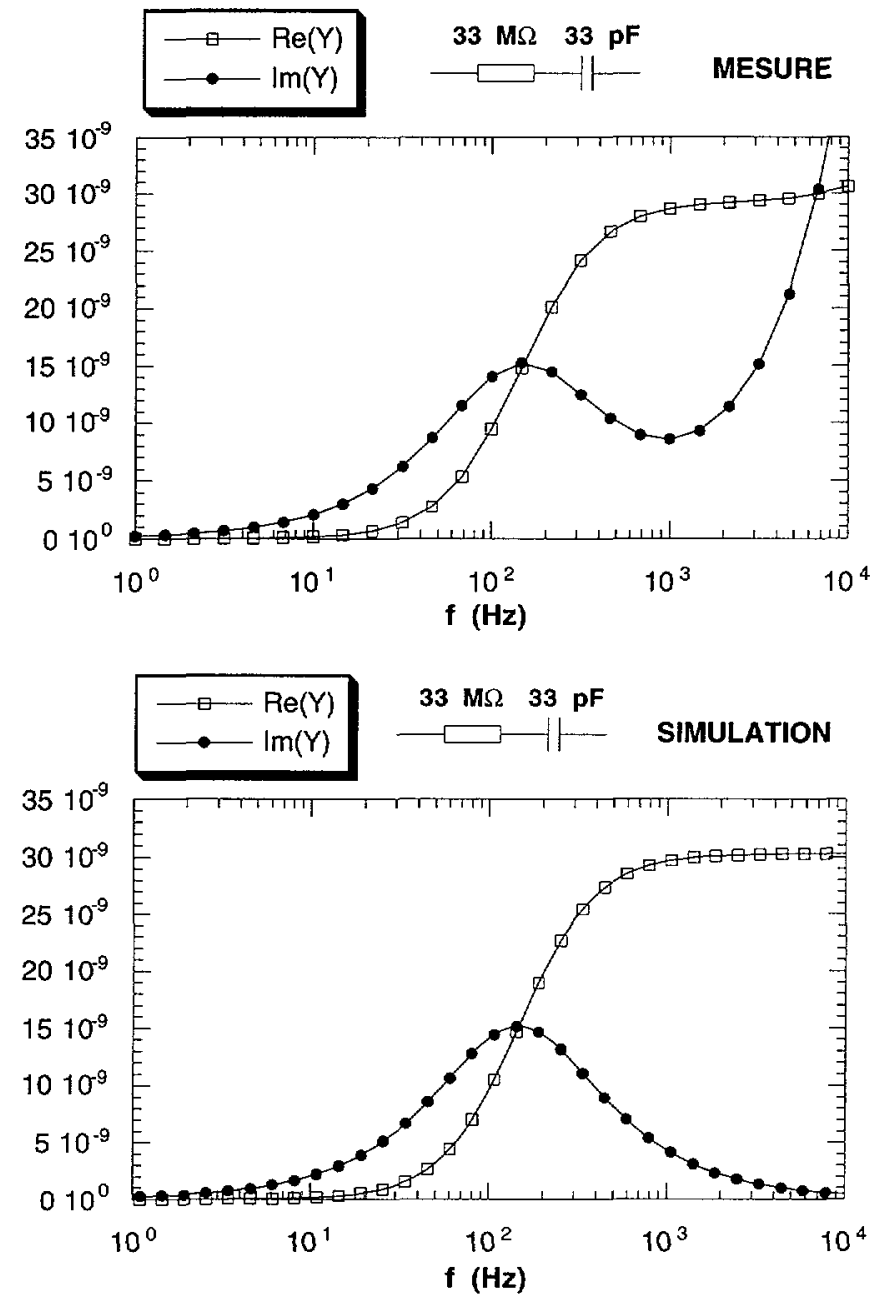

Fig. 8. - Admittance complexe en fonction de la fréquence pour un échantillon modèle. Limitation du système en haute fréquence : $R=33 \mathrm{M} \Omega, C=33 \mathrm{pF}$.

[Complex admittance vs. frequency for a model sample. High frequency limitation: $R=33 \mathrm{M} \Omega$. $C=33 \mathrm{pF}$.]

propriétés différentes sont susceptibles de se manifester à basse et à très basse fréquence. Les échantillons étudiés ont une épaisseur de $40 \mathrm{~mm}$. Les électrodes en $\mathrm{Ag} / \mathrm{Pd}$ sont sérigraphiées à la température ambiante puis recuites à $800^{\circ} \mathrm{C}$ pendant 1 heure. Des mesures effectuées pour deux formulations différentes sont présentées sur la figure 9 . L'évolution de l'impédance $Z^{*}(\omega)$ de ces matériaux est tracée dans le plan complexe où la partie imaginaire de $Z^{*}$ est représentée en fonction de la partie réelle, avec $\omega$ comme paramètre. Cette représentation a été retenue car elle est la plus employée dans la littérature sur ce type de matériaux. Expérimentalement des arcs de cercles décentrés par rapport à l'axe des réels avec un angle $\theta$ sont obtenus. Ce décalage est proportionnel au degré d'inhomogénéité de la varistance [16]. 


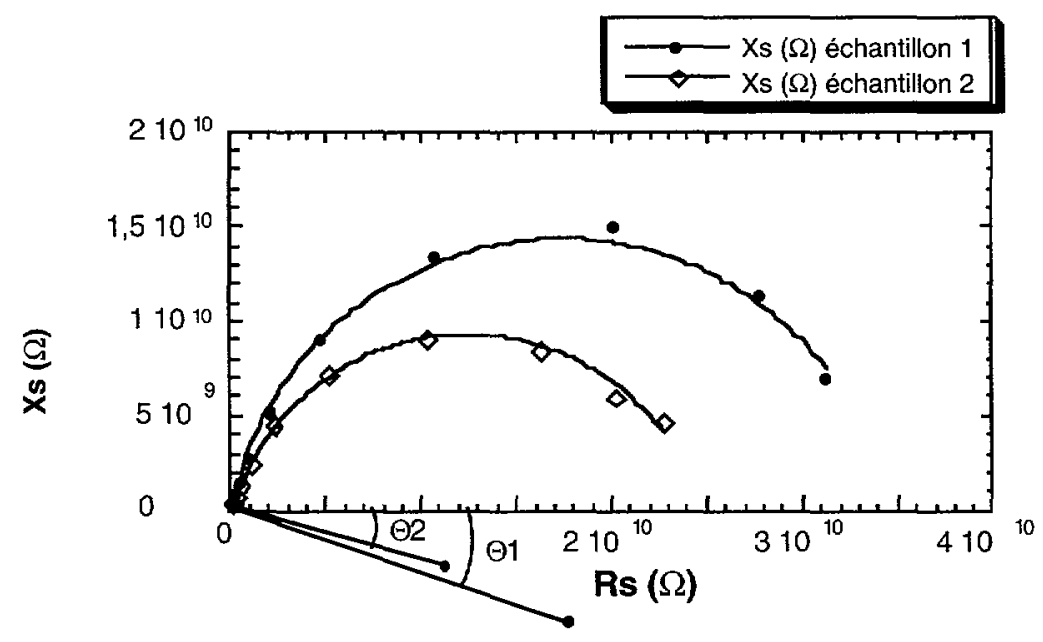

Fig. 9. - Spectres d'impédance complexe de deux varistances $\mathrm{ZnO}$ : influence de la formulation sur la fréquence de relaxation et l'angle $\theta$.

[Complex impedance spectra for two $\mathrm{ZnO}$ based varistors: influence of formulation on the relaxation frequency and $\theta$.]

Les points d'intersection de la courbe avec l'axe sont déterminés pour $f=\infty$ et $f=0$. De ces deux points, seul le dernier peut être obtenu expérimentalement, en appliquant aux bornes de l'échantillon étudié un échelon de tension et en mesurant le courant de conduction. Ces résultats montrent clairement l'existence de mécanismes de polarisation interfaciale dans ce type de matériaux. Il est encore plus remarquable que leur fréquence de relaxation et leur angle de décentrage, toutes deux caractéristiques de l'inhomogéneité du matériau, dépendent aussi nettement de la formulation des matériaux étudiés.

Ces résultats sont comparés à ceux obtenus à partir d'une mesure utilisant une méthode directe consistant à prélever les valeurs de la tension et du courant en fonction du temps pour diverses fréquences et en effectuant un échantillonnage de ces signaux. Cette méthode directe si elle permet des mesures dans une gamme plus étendue de fréquences (essentiellement les plus basses) ne présente pourtant pas une précision suffisante compte tenu de l'appareillage utilisé.

Les valeurs mesurées se situent en effet à la limite de leur sensibilité. Les influences externes, tel que le bruit, ne sont plus éliminées comme dans le cas des mesures effectuées à l'aide de l'analyseur de réponse en fréquence.

Cette comparaison, présentée sur la figure 10, nous permet toutefois de remarquer une bonne concordance entre les deux types de mesures. Les différences observées sont inférieures à $10 \%$, sauf pour la fréquence la plus élevée, sans qu'on puisse réellement incriminer notre dispositif. Hormis ces différences, un très bon alignement des points sur un arc de cercle passant par la valeur $R_{\mathrm{dc}}$ (déterminée avec une assez bonne précision par la mesure du courant de fuite sous tension continue) est obtenu. La méthode de mesure développée permet donc de caractériser de manière fiable le comportement basse fréquence de matériaux céramiques. Cette possibilité devrait permettre de confirmer certains travaux récents concernant le vieillissement des varistances sous chocs répétés pour lesquels la dérive du degré d'homogénéité - et donc de l'angle de décentrage - pourrait en effet être utilisé comme un excellent marqueur de leur comportement sous contraintes. 


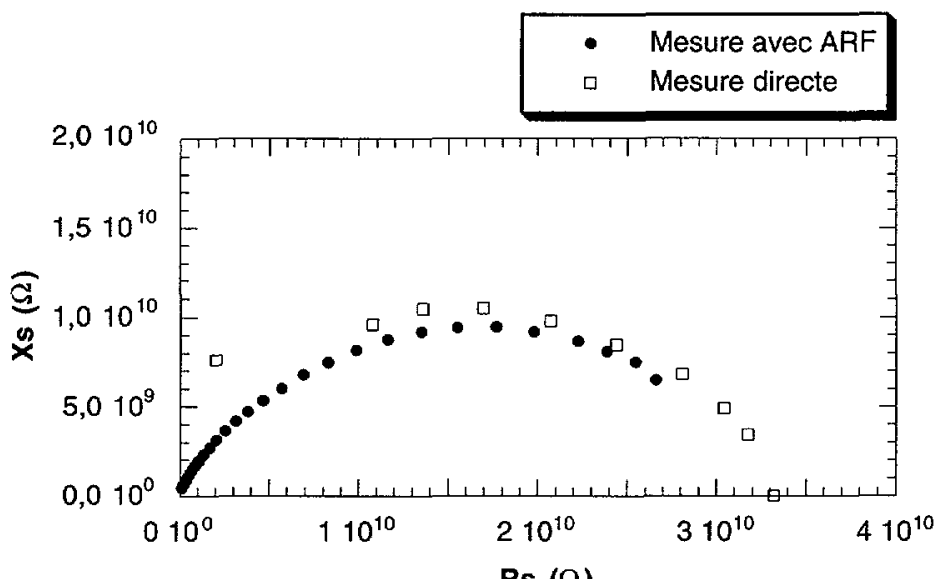

Fig. 10. - Comparaison entre les résultats des mesures directes et avec ARF pour une varistance $\mathrm{ZnO}$.

[Comparison between direct measurement and measurement using a FRA for $\mathrm{ZnO}$ based varistors.]

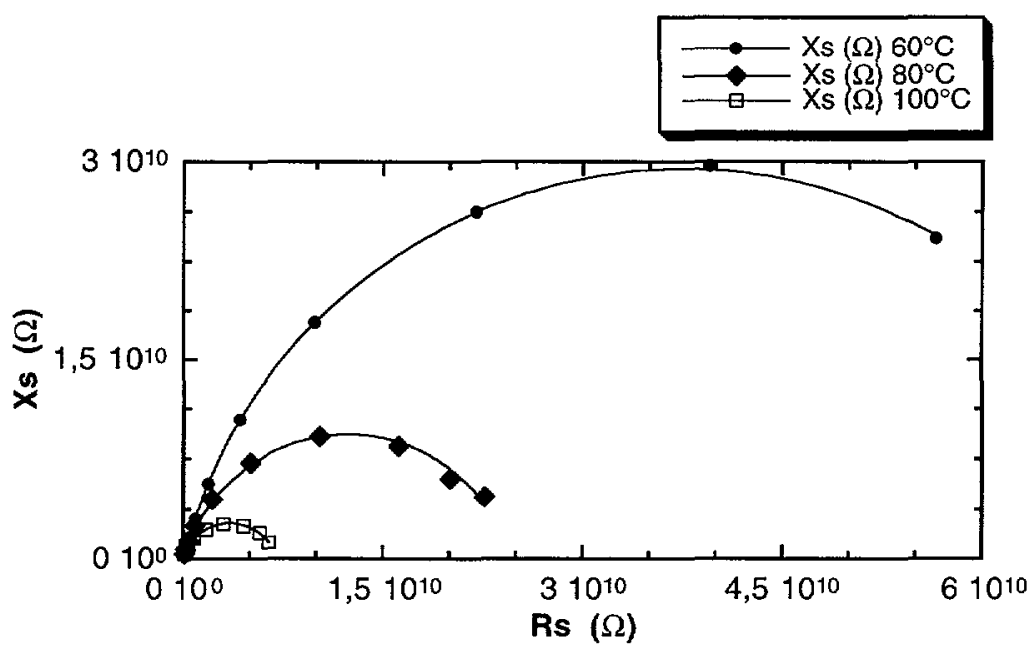

Fig. 11. - Spectres d'impédance complexe d'une varistance $\mathrm{ZnO}$ en fonction de la température.

[Complex impedance spectra for a $\mathrm{ZnO}$ based varistor vs. temperature.]

Notre dispositif permet aussi, par le suivi du comportement en température de la réponse fréquentielle, de déterminer l'énergie d'activation du processus de relaxation et par là même sa nature. Une telle étude a été menée et un exemple de résultat est présenté sur la figure 11. Nous n'entrerons pas davantage dans le détail de ces résultats car notre objectif est ici de démontrer la validité du système de mesure et non de nous lancer dans une étude fine de l'influence de la structure de ces matériaux sur la réponse fréquentielle. 


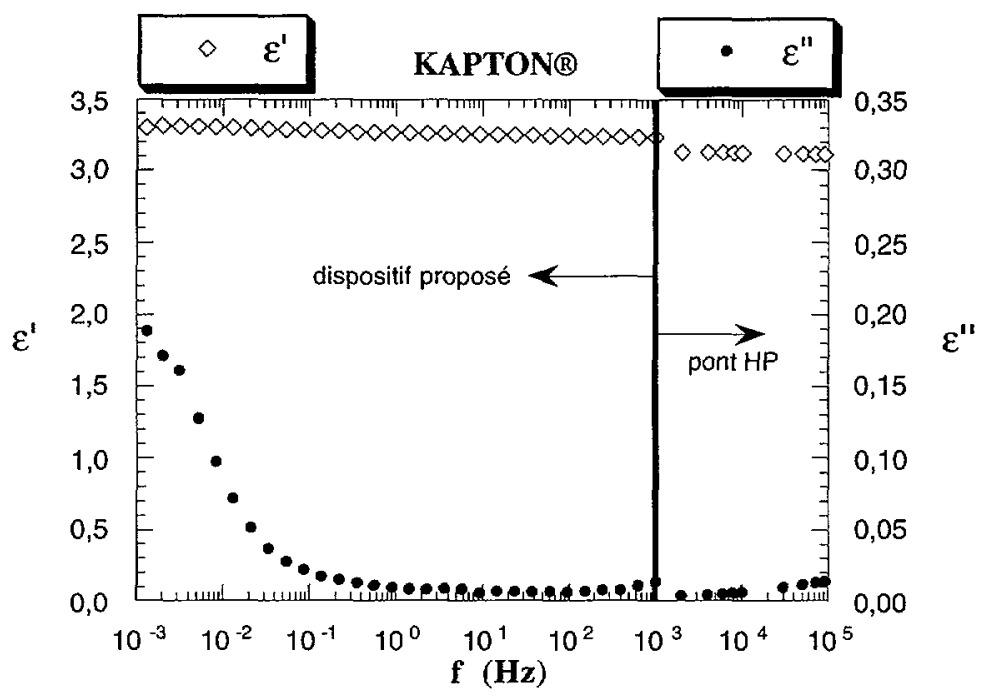

Fig. 12. - Spectre fréquentiel de $\varepsilon^{\prime}$ et $\varepsilon^{\prime \prime}$ pour le Kapton ${ }^{\circledR}$

[Frequency spectrum of $\varepsilon^{\prime}$ and $\varepsilon^{\prime \prime}$ for Kapton ${ }^{\circledR}$ ]

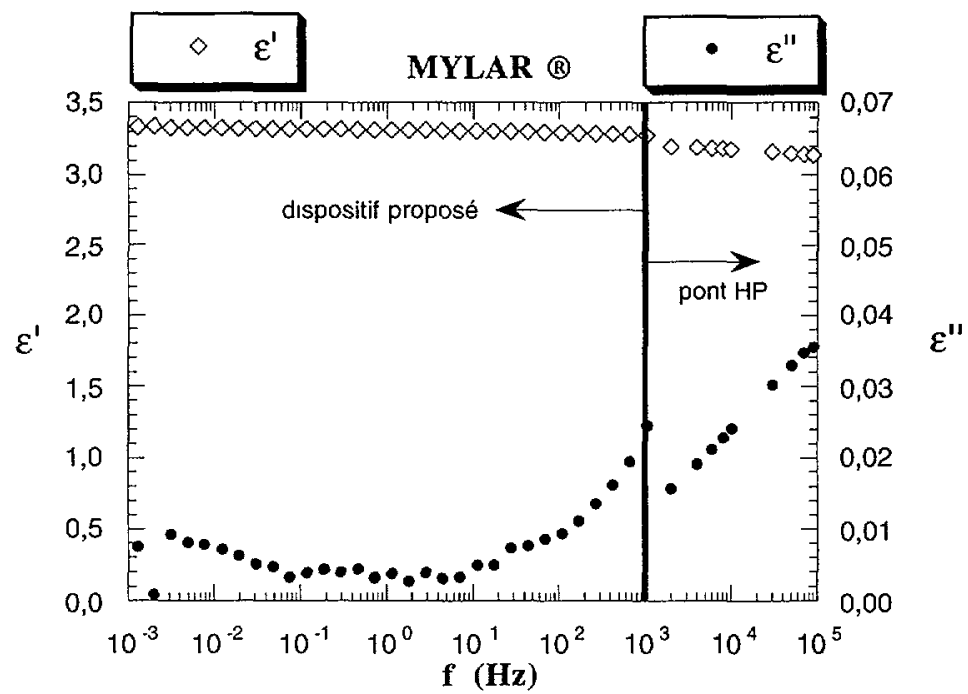

Fig. 13. - Spectre fréquentiel de $\varepsilon^{\prime}$ et $\varepsilon^{\prime \prime}$ pour le Mylar ${ }^{\circledR}$

[Frequency spectrum of $\varepsilon^{\prime}$ and $\varepsilon^{\prime \prime}$ for Mylar ${ }^{\circledR}$ ]

4.3. CaRACTÉRISATION DES MATÉRIAUX POLYMÈres. - Les matériaux polymères étudiés sont du même type que ceux généralement rencontrés dans l'isolation statorique des machines tournantes basse tension. Deux familles de polymères sont étudiées. Il s'agit d'un polyester, le Poly-Éthylène Terephtalate, et d'un polyimide, le Kapton. L'étude du comportement très basse fréquence de ces matériaux a été récemment considérée comme très importante pour assurer un suivi du vieillissement et donc la fiabilité des systèmes dans lesquels ils sont utilisés. En 
effet, quel que soit le mode d'alimentation des machines considérées ( $50 \mathrm{~Hz} \mathrm{AC}$ ou onduleurs à Modulation de Largeur d'Impulsions), la conclusion principale des études développées dans le cadre de la compréhension des mécanismes de vieillissement de l'isolation statorique, est que ceux-ci se traduisent par la création de charges libres $[17,18]$. Celles-ci proviennent de la dissociation extrinsèque ou intrinsèque des chaînes moléculaires. En migrant, ces charges vont contribuer, soit à augmenter la conductivité volumique de ces matériaux, soit à participer aux mécanismes de polarisation. Leur caractérisation à très basse fréquence apparaît donc nécessaire pour valider ces hypothèses. Les figures 12 et 13 décrivent le comportement fréquentiel des deux types de matériaux étudiés avant vieillissement. Les échantillons ont une épaisseur de $25 \mu \mathrm{m}$. Les électrodes en aluminium de $20 \mathrm{~mm}$ de diamètre sont déposées par évaporation thermique. Aucun mécanisme de polarisation interfaciale n'est détecté. Ceci n'est que peu surprenant compte tenu de la nature homogène des deux matériaux étudiés. Nous avons représenté sur les mêmes figures, les variations de permittivité dans la gamme de fréquence $10^{3}-10^{5} \mathrm{~Hz}$, mesures réalisées à l'aide d'un impédancemètre (HP 4284A). On observe une bonne correspondance des résultats obtenus par les deux techniques de mesure, tant sur les valeurs de $\varepsilon^{\prime}$ que de $\varepsilon^{\prime \prime}$. Le système proposé pourra donc être utilisé dans le cadre de l'étude développée dans notre laboratoire sur le vieillissement des isolations des machines tournantes.

\section{Conclusion}

La caractérisation très basse fréquence des matériaux diélectriques s'avère nécessaire pour la compréhension et la mise en évidence des phénomènes de relaxation, pour l'analyse fine de la structure et de l'homogénéité de certains matériaux et pour le suivi et la modélisation des mécanismes de vieillissement. Elle n'avait jamais pu, jusqu'à maintenant, être réalisée de manière directe sur des matériaux diélectriques solides à faibles pertes tels que ceux généralement rencontrés en Génie Électrique. Le dispositif mis au point dans notre laboratoire permet désormais cette caractérisation. Différents matériaux ont été testés et les résultats obtenus sont satisfaisants. Ce système va maintenant être utilisé dans les travaux que nous développons dans le domaine général du vieillissement des matériaux diélectriques solides.

\section{Bibliographie}

[1] Nordik E., Reclassement des hydroalternateurs, Electra 162 (1995) 86-94.

[2] Ramu T., Degradation of H.V generator insulation under mechanical, electrical and thermal stresses, Proc. IEEE Intem. Symp. Elect. Ins. (1990) 21-24.

[3] Hedvig P., Dielectric Spectroscopy of Polymers, Adam Hilger Ltd Ed. (Bristol, 1977).

[4] Takada T., Space charge formation in dielectrics, IEEE Trans, elect. ins. 21 (1986) 873879.

[5] Lebey T. and Laurent C., Charge injection and electroluminescence as a prelude to dielectric breakdown, J. Appl. Phys. 67 (1990) 275-282.

[6] Japan IEE. Tech. Rept., Thermally stimulated current in electrical insulation materials, $\mathrm{n}^{\circ} 194$ (1985).

[7] Berthier S., Optique et matériaux composites, Polytechnica Ed. (Paris, 1993).

[8] Bosma T., Lebey T., Pouillès V., Chênerie I. and Bui Ai, The use of mixing laws for modelling climatic ageing of dielectric materials, J. Phys. D : Appl. Phys. 28 (1995) 11801186. 
[9] Pouillès V., Chênerie I., Bollioli S. et Lebey T., Cellule microbande en anneau pour la caractérisation diélectrique dans la bande UHF, Journées de Caractérisation des Matériaux en Micro-Ondes (Brest, octobre 1994) pp. 47-51.

[10] MacDonald J.R., Impedance Spectroscopy, J. Wiley \& Sons (New York, 1987).

[11] Sugget A., Time domain methods, Dielectrics and related molecular processes, vol. 1, The chemical society (London, 1972).

[12] Jonscher A.K., Dielectric relaxation in solids, Chelsea dielectrics press (London, 1983).

[13] Li H.-M., Fouracre R. and Criton B., Some considerations for obtaining the low frequency response of dielectrics from absorption current measurements, Proc. Conf. Elec. Insul. Diel. Phen. (CEIDP) (1994) 238-244.

[14] Morse C.T., A computer controlled apparatus for measuring AC properties of materials over the frequency range $10^{-5}$ to $10^{5} \mathrm{~Hz}, J$. Phys. E : Scient. Instr. 7 (1974) 470-482.

[15] Burr Brown, Integrated Circuits Data Handbook (1995).

[16] Laffargue J.M., Caractérisation de la dégradation des varistances à base d'oxyde de zinc par la spectroscopie d'impédance, Thèse UPS (Toulouse, 1995).

[17] Pouillès V., Lebey T. and Bui Ai, Correlation between energy dissipation and permittivity in dielectric material ageing studies, Proc. Int. Symp. on High Volt. Eng., paper 1022 (1995).

[18] Goffaux R., Contribution à la caractérisation de l'isolation entre spires des bobinages statoriques des machines à courant alternatif, R.G.E. 91 (1991) 1-14. 